\title{
Applications of Artificial Neural Network Modeling in Drug Discovery
}

\section{Feng Cheng and Vijaykumar Sutariya*}

College of Pharmacy, University of South Florida, 12901 Bruce B. Downs Blvd., MDC 30, Tampa, Florida 33612-4749, USA

Artificial Neural Networks (ANNs) modeling is a group of computer algorithms for modeling and pattern recognition, functioning similarly to the neurons of the brain. The brain learns from its experience. In the brain, a biological neuron receives inputs from many external resources, combines them, performs a non-linear operation, and then makes a decision based on the final results. The ANNs are a type of mathematical model that simulates the biological nervous system and draws on analogues of adaptive biological neurons. A major advantage of ANNs compared to statistical modeling is that they do not require rigidly structured experimental designs and can map functions using historical or incomplete data. ANNs are good recognizers of patterns and robust classifiers, with the ability to generate when making decisions based on imprecise input data.

ANNs are known to be a powerful tool to simulate various nonlinear systems and have been applied to numerous problems of considerable complexity in many field including pharmaceutical research, engineering, psychology and medicinal chemistry.

The potential applications of ANN methodology in the pharmaceutical sciences are broad. In this paper, we will review some applications of ANNs in drug discovery.

\section{Quantitative Structure-Activity Relationship (QSAR)}

QSAR correlates physicochemical parameters of compounds with chemical or biological activities. These parameters include topological parameters, molecular weight, molar volume, electronegativity, $\log \mathrm{P}$, hydrogen acceptor, hydrogen donor and molar refractivity. ANNs have been shown to be an effective tool to establish this type of relationship and predict the activities of new compounds. Jaen-Oltra et al. [1] developed a new topological method to predict antimicrobial properties of quinolones derivatives on the basis of their chemical structures. An ANN with suitable set of topological descriptors and training algorithms was used to determine the minimum inhibitory concentration of quinolones. In another example, $\mathrm{Hu}$ et al. [2] combined ANNs, quantum chemistry and molecular docking methods to design novel Aldose Reductase Inhibitors (ARIs). Physicochemical parameters including electronegativity and molar volume of known inhibitors were calculated using quantum chemistry methods. Using these parameters as input nodes, an ANN based QSAR model was constructed and the biological activities of new compounds were predicted. The further molecular docking analysis showed all the predicted potent compounds by ANNs binded well to the active site of the aldose reductase.

\section{Virtual Screening (VS)}

Virtual screening is a novel approach to speed the drug discovery process. It applies computational methods to quickly search (or "screen") compounds with known chemical structures to identify the compounds with high biological activities in a compound database. ANNs-based QSAR models are widely chosen as the prediction methods in the virtual screening. Recently, Myint et al. [3] have developed a 2D fingerprint-based artificial neural network QSAR (FANN-QSAR) method for a large scale drug virtual screening. A set of 1699 cannabinoid receptor subtype 2 (CB2) ligands with reported activities was used for the model training and a five-fold crossvalidation. A 2D circular topological fingerprint, ECFP6, was chosen as the neural network input. Using this ECFP6-ANN-QSAR model, they predicted GPCR cannabinoid ligand binding affinities of a large $\mathrm{NCI}$ compound database with over 200,000 structurally diverse compounds. Two compounds with nanomolar activities were discovered.

\section{Quantitative Structure Toxicity Relationship (QSTR)}

ANNs were also widely used in pharmacotoxicology, especially in QSTR studies. QSTR is a mathematical relationship between the chemical's quantitative molecular descriptors and its toxicological activities. QSTR models can be applied to predict the toxicity of compounds. Similar to QSAR, the molecular descriptors of QSTR are derived from physicochemical properties of the compounds. These descriptors are then correlated with a toxicological response of interest through ANNs modeling. For example, Gao et al. [4] developed an ANN-based prediction model of the bio-toxicity of substituted benzene. In this study, 78 substituted benzene compounds were chosen to establish and validate ANN-QSTR models. Five topological indexes were chosen as input nodes of network and the optimum network was 5-17-1. The ANN-QSTR model was validated by 23 substituted benzene compounds. The correlation coefficient between predicted and actual toxicological activities of these compounds was found to be 0.9088 .

\section{Pharmacokinetic and Pharmacodynamics}

The ANN technology offers an exciting alternative to monitor complex interactions between drug substance and physiological system that are usually monitored by pharmacodynamics. Haider et al. [5] reported on a predictive PK/PD model for an oral hypoglycemic agent (repaglinide) using ANNs. They concluded that ANNs were a quick and simple method for predicting and identifying significant covariates.

Gobburu et al. [6] also applied an ANN to PK/PD analysis and concluded that it is a versatile computational tool and exhibits clear advantage over conventional model-independent PK/PD analysis. Chow et al. [7] compared the predictive ability of ANNs with that of NNMEM for tobramycin plasma levels in pediatric patients and conclude that ANNs have the potential to become a useful analytical tool for population pharmacokinetic data analysis. Ritschel et al. [8] used ANNs to predict human pharmacokinetics parameters (total clearance and distribution volume) from a combined data set of physicochemical properties of drugs (protein binding, partition coefficient, dissolution constant) and animal pharmacokinetic parameters (total clearance and distribution volume).

*Corresponding author: Vijaykumar Sutariya, College of Pharmacy, University of South Florida, 12901 Bruce B Downs Blvd., MDC 3012 Tampa, Florida 33612-4749, USA, Tel: 813-974-1401; Fax: 813-974-9890; E-mail: vsutariy@health.usf.edu

Received October 30, 2012; Accepted November 01, 2012; Published November 04, 2012

Citation: Cheng F, Vijaykumar S (2012) Applications of Artificial Neural Network Modeling in Drug Discovery. Clin Exp Pharmacol 2:e113. doi:10.4172/2161 1459.1000e113

Copyright: $\odot 2012$ Cheng F, et al. This is an open-access article distributed under the terms of the Creative Commons Attribution License, which permits unrestricted use, distribution, and reproduction in any medium, provided the original author and source are credited. 
Citation: Cheng F, Vijaykumar S (2012) Applications of Artificial Neural Network Modeling in Drug Discovery. Clin Exp Pharmacol 2:e113. doi:10.4172/2161-1459.1000e113

\section{Conclusion and Perspective}

ANNs are powerful tools for drug discovery. The utility of ANNs in the pharmaceutical field has recently gained enormous attention due to their ability to solve problems involving complex pattern recognition. We believe the application of ANNs in drug discovery will have the potential for further development.

\section{References}

1. Jaen-Oltra J, Salabert-Salvador MT, Garcia-March FJ, Perez-Gimenez F, Tomas-Vert F (2000) Artificial neural network applied to prediction of fluorquinolone antibacterial activity by topological methods. J Med Chem 43: 1143-1148.

2. Hu L, Chen G, Chau RM (2006) A neural networks-based drug discovery approach and its application for designing aldose reductase inhibitors. J Mol Graph Model 24: 244-253.

3. Myint KZ, Wang L, Tong Q, Xie XQ (2012) Molecular fingerprint-based artificial neural networks QSAR for ligand biological activity predictions. Mol Pharm 9: 2912-2923.

4. Gao DW, Wang P, Liang H, Peng YZ (2003) A study on prediction of the biotoxicity of substituted benzene based on artificial neural network. J Environ Sci Health B 38: 571-579.

5. Haidar SH, Johnson SB, Fossler MJ, Hussain AS (2002) Modeling the pharmacokinetics and pharmacodynamics of a unique oral hypoglycemic agent using neural networks. Pharm Res 19: 87-91.

6. Gobburu JV, Chen EP (1996) Artificial neural networks as a novel approach to integrated pharmacokinetic-pharmacodynamic analysis. J Pharm Sci 85: 505510.

7. Chow HH, Tolle KM, Roe DJ, Elsberry V, Chen H (1997) Application of neural networks to population pharmacokinetic data analysis. J Pharm Sci 86: 840845.

8. Ritschel WA, Akileswaran R, Hussain AS (1995) Application of neural networks for the prediction of human pharmacokinetic parameters. Methods Find Exp Clin Pharmacol 17: 629-643. 